\title{
Preseeding with autologous fibroblasts improves endothelialization of glutaraldehyde-fixed porcine aortic valves
}

\author{
Helmut Gulbins, MD \\ Angelika Goldemund \\ Ingrid Anderson \\ Ulrike Haas \\ Antje Uhlig \\ Bruno Meiser, MD \\ Bruno Reichart, MD
}

From the Department of Cardiac Surgery,
University Hospital Grosshadern, Munich,
Germany.

Received for publication April 12, 2002; revisions requested May 30, 2002; revisions received July 2, 2002; accepted for publication July 8, 2002.

Address for reprints: Helmut Gulbins, MD, Department of Cardiac Surgery, University Hospital Grosshadern, LMU Munich, D-81366 Munich, Germany (E-mail: H.Gulbins@hch.med.uni-muenchen.de).

J Thorac Cardiovasc Surg 2003;125: 592-601

Copyright (C) 2003 by The American Association for Thoracic Surgery

$0022-5223 / 2003 \$ 30.00+0$

doi: $10.1067 / \mathrm{mtc} .2003 .48$
Objective: This study represents the development of a treatment and seeding procedure to improve endothelial cellular adhesion on glutaraldehyde-fixed valves.

Methods: Porcine aortic valves were fixed with $0.2 \%$ glutaraldehyde. Wall pieces of these valves had either no additional treatment $(n=4)$, incubation in M199 Earle $(1 \times)$, with sodium carbonate at $2.2 \mathrm{~g} / \mathrm{L}$ without $\mathrm{L}$-glutamine for 24 hours $(\mathrm{n}=4)$, or additional pretreatment with $5 \%, 10 \%$, or $15 \%$ citric acid (three groups, $\mathrm{n}=4$ each). Thereafter the pieces were washed and buffered to a physiologic $\mathrm{pH}$. This was followed by seeding of human endothelial cells $\left(5 \times 10^{6}\right.$ cells $)$. On the basis of the results of these pilot tests, complete glutaraldehyde-fixed aortic roots treated with $10 \%$ citric acid were subjected to cell seeding. The valves were seeded with endothelial cells $\left(4.3 \times 10^{6}\right.$ cells $)$ either alone $(n=4)$ or in combination with preseeding of autologous fibroblasts $\left(2.4 \times 10^{7}\right.$ cells, $\left.n=4\right)$. After each seeding procedure specimens of the free wall of the grafts were taken. In addition, one leaflet was taken for histologic examination after endothelial cell seeding, after 7 days, and after 21 days. Finally, two commercially available stentless aortic valve prostheses (Freestyle; Medtronic, Inc, Minneapolis, Minn) were treated with $10 \%$ citric acid and seeded with human fibroblasts and endothelial cells. Specimen were taken according to the glutaraldehyde-fixed aortic roots. Specimen of all experiments were examined with scanning electron microscopy. Frozen sections were stained immunohistochemically for collagen IV, factor VIII, and CD31.

Results: On untreated glutaraldehyde-fixed aortic wall pieces, only poor adhesion $(24 \%)$ was seen. No viable cells were found after 1 week. Cellular adhesion was best on aortic wall pieces pretreated with $10 \%$ citric acid. After 7 days, the cells formed a confluent layer. Endothelial cell seeding on citric acid-treated complete aortic valves showed $45 \%$ adhesion, but no confluent layer was found after 1 week. Preseeding of these valves with autologous fibroblasts resulted in an endothelial cellular adhesion of $76 \%$ and a confluent endothelial cell layer after 7 days. The layer remained stable for at least 21 days. Results of staining for collagen IV, factor VIII, and CD31 were positive on the luminal side of these valves, indicating the synthesis of matrix proteins and viability of the cells. Pretreatment of commercially available porcine valves with $10 \%$ citric acid and preseeding with autologous fibroblasts followed by endothelial cell seeding resulted in an adhesion of $78 \%$. The cells formed a confluent cell layer after 7 days.

Conclusions: Pretreatment of glutaraldehyde-fixed porcine aortic valves with citric acid established a surface more suitable for cellular attachment. Preseeding these valves with autologous fibroblasts resulted in a confluent endothelial cell layer on 
the luminal surface. Flow tests and animal experiments are necessary for further assessment of durability and shear stress resistance.

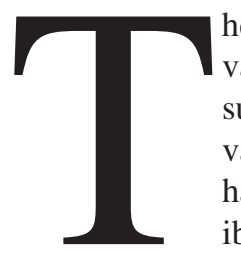

he degeneration of glutaraldehyde-fixed valves, with subsequent calcification and tissue failure, is the main disadvantage of these valvular prostheses. Different strategies have been developed to increase biocompatibility and durability. ${ }^{1-4}$ Covering the surface of porcine glutaraldehyde-fixed valve prostheses with autologous endothelial cells (ECs) of the recipient may delay valve degeneration and further reduce thromboembolic events. However, a durable EC lining has been established with only limited success on commercially available glutaraldehyde-fixed aortic valves. ${ }^{5-12}$ The purpose of our study was to develop a new treatment procedure for these valve prostheses, enabling seeding of viable human ECs to cover the entire luminal surface. An additional goal was to achieve a confluent EC layer that would remain stable for at least 21 days under culture conditions.

\section{Material and Methods \\ Valves and Cells}

Porcine valve-containing aortic roots were dissected from healthy Landrace pigs. The hearts were obtained from the local slaughterhouse. After preparation, the miniroots were incubated in $0.2 \%$ glutaraldehyde, buffered with phosphate-buffered saline solution (Dulbecco buffer without calcium and magnesium ion; Biochrom AG, Berlin, Germany) for at least 7 days. For the first dish experiments, aortic wall pieces with a surface of $4 \mathrm{~cm}^{2}$ were taken. For the seeding experiments, complete valve-containing miniroots were used.

Human ECs and fibroblasts were isolated from saphenous vein pieces as described previously elsewhere. ${ }^{13-15}$ In brief, human saphenous vein pieces with a mean length of $9.8 \pm 4.6 \mathrm{~cm}$ (range $4.1-25 \mathrm{~cm}$ ) were stored in medium M199 Earle $(1 \times)$, with sodium carbonate at $2.2 \mathrm{~g} / \mathrm{L}$ without L-glutamine (M-199; Biochrom). The vein pieces were left over from routine aortocoronary bypass operations, and the patients had given their informed consent that these pieces could be used in the laboratory. The local ethic committee approved the anonymous use of the saphenous vein pieces for this experimental study. The vein pieces were cannulated, rinsed with buffered medium, and incubated with $0.1 \%$ collagenase (Worthington Biochemical Corporation, Lakewood, $\mathrm{NJ}$ ) for 20 minutes at $37^{\circ} \mathrm{C}$ with $5 \%$ carbon dioxide. Thereafter the collagenase reaction was stopped and the cell suspension was centrifuged at $1000 \mathrm{rpm}$ for 10 minutes. The cell pellet was resuspended in EC growth medium (PromoCell GmbH, Heidelberg, Germany) and plated on culture dishes. For isolation of the fibroblasts, the same vein pieces were again filled with $0.1 \%$ collagenase and incubated for 30 minutes. This procedure was repeated twice. Again, the cell suspension was centrifuged at 1000 rpm for 10 minutes, the pellet was resuspended in fibroblast growth medium (PromoCell), and the resultant suspension was plated on culture dishes. For passaging, both cell types were digested with trypsin after reaching confluence and then centrifuged, resuspended in cell medium, and plated again. For cell counting, $30 \mu \mathrm{L}$ of the resuspended cell suspension was incubated with trypan blue and counted with a Neubauer chamber.

\section{Culture Dish Experiments With Porcine Aortic Wall Pieces}

Twenty aortic wall pieces of $4 \mathrm{~cm}^{2}$ were divided into five groups ( $n=4$ each group). Group A had no treatment after glutaraldehyde fixation. All pieces of the following four groups were incubated with M-199 for 24 hours at $4^{\circ} \mathrm{C}$. Group B had no further treatment, and groups $\mathrm{C}, \mathrm{D}$, and $\mathrm{E}$ were then incubated with citric acid at concentrations of $5 \%, 10 \%$, and $15 \%$, respectively. This was followed by washing with distilled water until a neutral $\mathrm{pH}$ was reached. The pieces then were put into 6-well culture dishes, all of which were incubated with $5 \times 10^{6}$ ECs. After 24 hours the pieces were cut in half and one part was examined with the scanning electron microscope. The second part of each piece was kept under culture conditions for 7 days, after which these pieces were also examined under the scanning electron microscope.

For assessment of possible toxic effects of glutaraldehyde released from the glutaraldehyde-fixed valves, the different procedures were tested in five groups $(\mathrm{n}=3$ each). Group A1 had no pretreatment but washing in $1000 \mathrm{~mL}$ physiologic sodium chloride solution for 10 minutes; all other groups had incubation with M-199 for 24 hours. Group B1 had no further treatment, and groups $\mathrm{C} 1, \mathrm{D} 1$, and $\mathrm{E} 1$ were treated with citric acid at concentrations of 5\%,10\%, and 15\%, respectively. All these pieces were put into culture dishes onto a confluent EC layer. After an incubation time of 72 hours, the dishes were examined for any changes within the ECs.

\section{Cell Seeding on Aortic Miniroots}

According to the results of the culture dish experiments, 12 glutaraldehyde-fixed aortic miniroots were divided into three groups of four valves each. Group 1 had no pretreatment but was washed in physiologic sodium chloride solution for 10 minutes. Groups 2 and 3 were incubated with M-199 for 24 hours. Group 3 valves underwent additional pretreatment with $10 \%$ citric acid for 5 minutes followed by washing with distilled water until a neutral $\mathrm{pH}$ was reached. The miniroots were then put into the cylinder of our seeding device (Figure 1). Two valves of each group were seeded with only ECs, whereas the others were preseeded with autologous fibroblasts followed by EC seeding.

For fibroblast seeding the device was filled with fibroblast growth medium and a cell suspension containing $2.4 \times 10^{7}$ fibroblasts on average (range 1.2-4.1 $\times 10^{7}$ cells). The seeding procedure lasted for a period of 24 hours. Every 30 minutes the device rotated for 150 seconds, stopping in a different position for the 


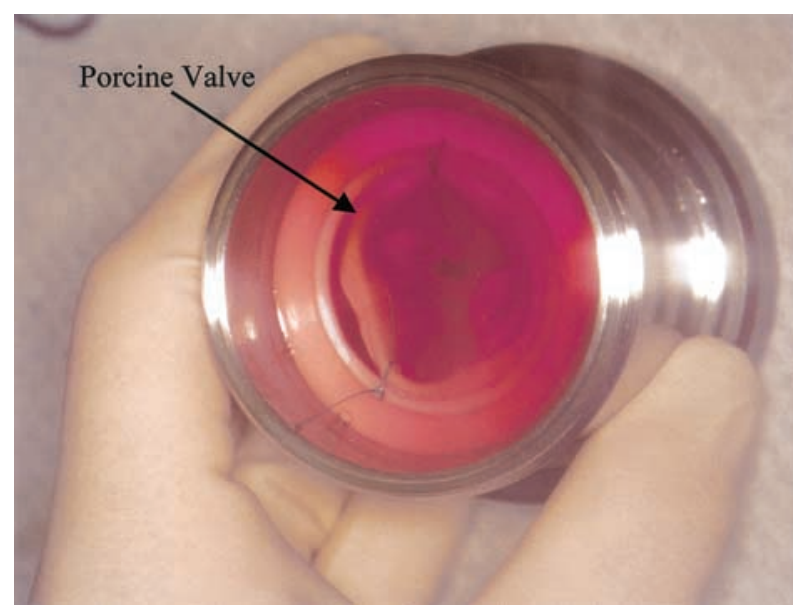

Figure 1. Cell seeding device. Valve is fixed in cylinder so that all parts are in contact with cell suspension. During seeding procedure, cylinder is rotated every $\mathbf{3 0}$ minutes to expose different parts of valve to cell seeding.

next resting period to ensure that all parts of the aortic roots were exposed to cell seeding. After the seeding procedure, the valves were kept under culture conditions for 7 days, followed by EC seeding. EC seeding was done analogously to fibroblast seeding. The average number of ECs was $4.3 \times 10^{7}$ (range 1.8-6 $\times 10^{7}$ cells). The first specimen for scanning electron microscopic examination and immunohistochemical staining was taken directly after the seeding procedure. One leaflet of each valve and one wall piece of the sinus of Valsalva were taken directly after EC seeding, the second specimen was taken 7 days after EC seeding, and the last was taken 21 days after EC seeding.

\section{Cell Seeding on Commercially Available Porcine Aortic Valve Prostheses}

Three commercially available stentless porcine aortic valve prostheses (Freestyle 21 and $23 \mathrm{~mm}$; Medtronic, Inc, Minneapolis, Minn) were put into the seeding device analogously to the valvecontaining aortic roots. The valves were incubated in M-199 for 24 hours at $4^{\circ} \mathrm{C}$ and pretreated with $10 \%$ citric acid for 5 minutes. After washing with distilled water until a neutral $\mathrm{pH}$ was reached, the valves were preseeded with fibroblasts, followed by EC seeding as described previously. Cell numbers were $1.9 \times 10^{7}, 2.2 \times$ $10^{7}$, and $2.8 \times 10^{7}$ cells for the fibroblast seeding. Specimens of the free aortic wall were taken directly after the seeding procedure and 7 days later. ECs were then seeded on these valves as described previously. The cell numbers were $1.1 \times 10^{8}, 3.9 \times 10^{7}$, and $2.2 \times 10^{7}$ cells. One leaflet of each valve and one wall piece of the sinus of Valsalva were taken directly after EC seeding, the second specimen was taken 7 days after EC seeding, and the last was taken 21 days after EC seeding.

\section{Immunohistochemical Staining}

Specimens of the aortic wall and valve leaflets were frozen and stored in liquid nitrogen. Eight-micrometer sections were stained with monoclonal antibodies against factor VIII, CD31 (DAKO
TABLE 1. Results of the dish experiments

\begin{tabular}{lccc}
\hline Group & EC survival & Confluent EC layer after 7 d & Cell death \\
\hline A & Neg & Neg & \\
B & Pos & Neg & \\
C & Pos & Pos & \\
D & Pos & Pos & \\
E & Pos & Neg & \\
A1 & & & Strongly pos \\
B1 & & & Neg \\
C1 & & & Neg \\
D1 & & & Neg \\
E1 & & & Weakly pos \\
\hline
\end{tabular}

Diagnostika GmbH, Hamburg Germany), and collagen IV (Sigma, Deisnhofen, Germany). For factor VIII staining a polyclonal antibody against antirabbit immunoglobulin G (Immundiagnostik, Bensheim, Germany) was used. Collagen IV and CD31 stainings were counterstained with a polyclonal antibody against mouse immunoglobulin G (CHEMICON International, Inc, Temecula, Calif). The results were validated with negative control stainings without the primary antibody. For confirmation of the immunofluorescence results, additional peroxidase staining was performed. After incubation with the primary antibodies against factor VIII, CD31, and collagen IV, an antimouse immunoglobulin G antibody (DAKO, Hamburg, Germany) was added and stained with 3-amino-9-ethylcarbazole (AEC; DAKO). Negative control preparations were done analogously to the fluorescence staining.

\section{Scanning Electron Microscopy}

Only cell layers with the typical cobblestone morphologic characteristics were accepted as EC layers. For analysis 10 visual fields of each specimen were evaluated under $1000 \times$ magnification. Of the leaflets, both fibrosal and ventricularis surfaces were evaluated. EC covering was assessed semiquantitatively by two examiners and classified as $100 \%, 75 \%, 50 \%$, or $25 \%$. A mean value of $95 \%$ or greater was thought to represent a confluent EC layer.

\section{Results}

Dish Experiments With Porcine Aortic Wall Pieces

EC seeding on glutaraldehyde-fixed aortic wall pieces resulted in sporadic surviving ECs on the surface (group A, Table 1). These cells completely disappeared 7 days after the cell seeding. No viable cells were found on the ground of the culture dish. Incubation with M-199 for 24 hours before EC seeding resulted in scattered cells on the surface of the aortic wall pieces (Figure 2). These cells also disappeared within 7 days. Nevertheless, on the bottoms of these culture dishes viable ECs had grown to confluence within this time.

Citric acid pretreatment at concentrations of 5\% and $10 \%$ improved the initial result of EC seeding. The aortic wall pieces of both groups showed a confluent EC layer after 7 days (Figure 3 ). Those pieces incubated with $15 \%$ citric acid had only scattered ECs after cell seeding. Seven days after 


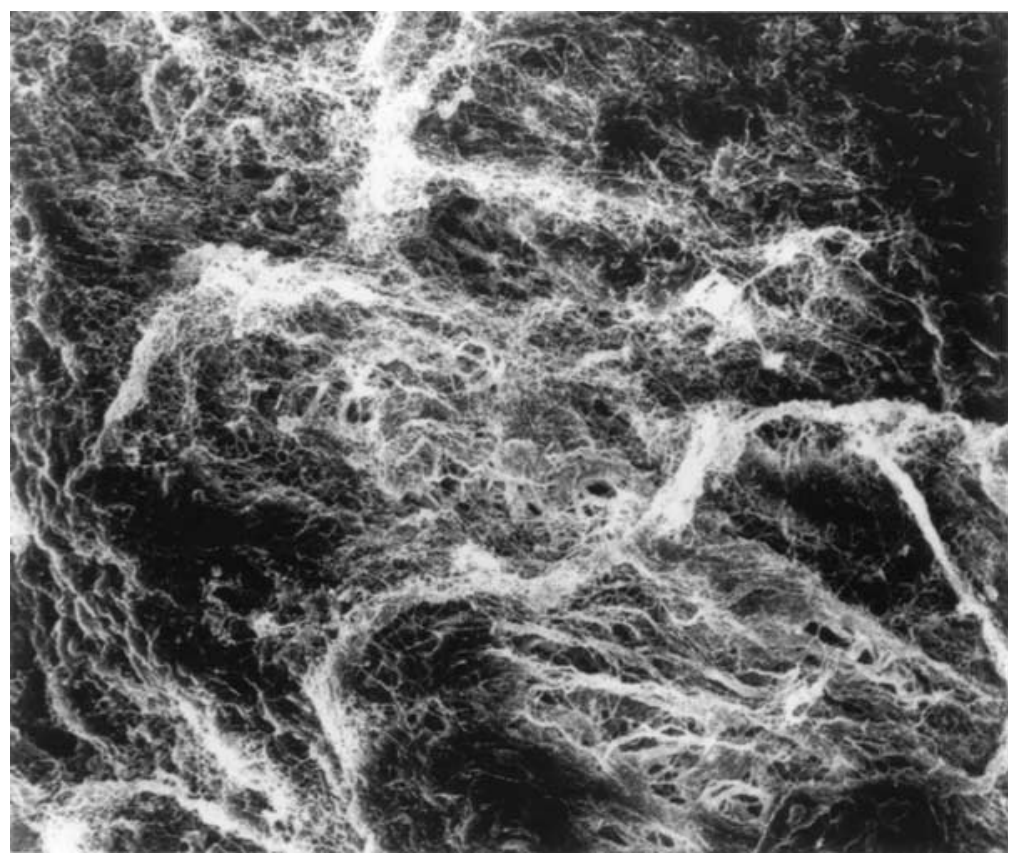

Figure 2. Aortic wall piece, glutaraldehyde fixed and incubated with M-199 for 24 hours, 7 days after EC seeding. No viable cells were observed under scanning electron microscopy (original magnification $500 \times$ ).

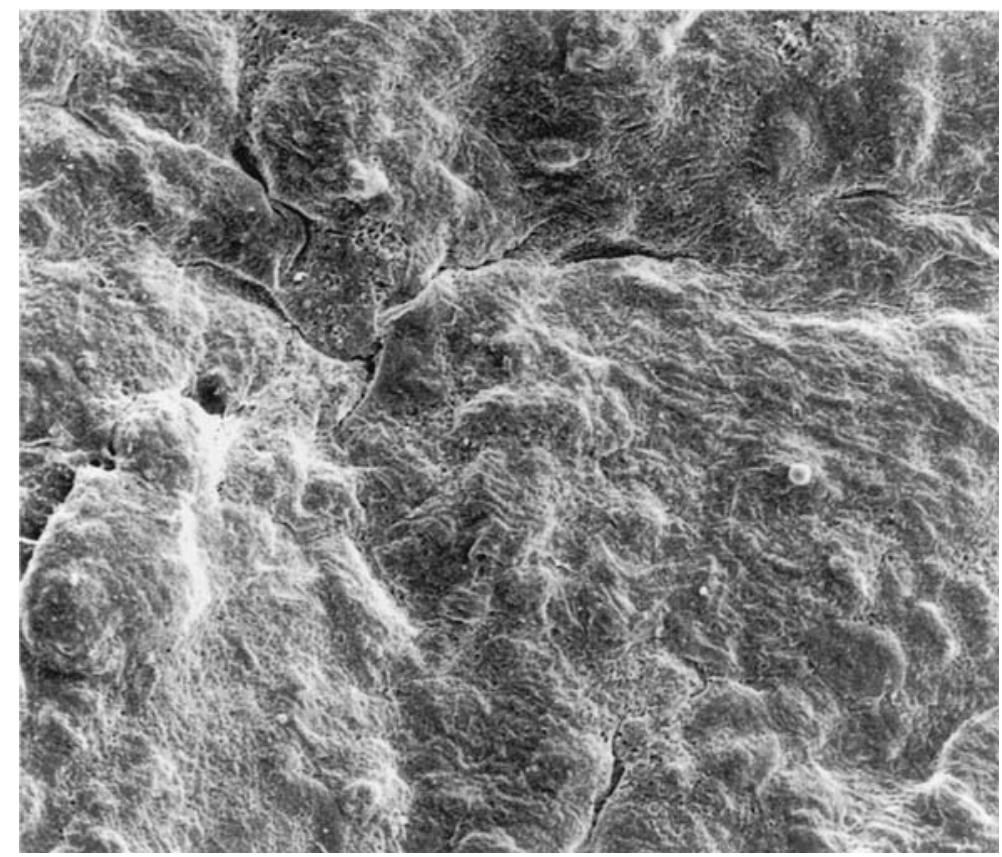

Figure 3. EC seeding on glutaraldehyde-fixed aortic wall piece after pretreatment with $5 \%$ citric acid after 7 days under culture conditions. Confluent EC layer is seen under scanning electron microscopy (original magnification $500 \times)$.

that only islands of connected ECs were seen without reaching confluence.

In the toxicity testing glutaraldehyde-fixed aortic wall pieces (group A1; Table 1) had a marginal zone without viable cells of $2.1 \pm 0.7 \mathrm{~cm}$ surrounding them after 72 hours of incubation (Figure 4, A). On the remaining culture dish, however, a confluent EC layer was seen. Glutaraldehydefixed aortic wall pieces after incubation with M-199 for 24 

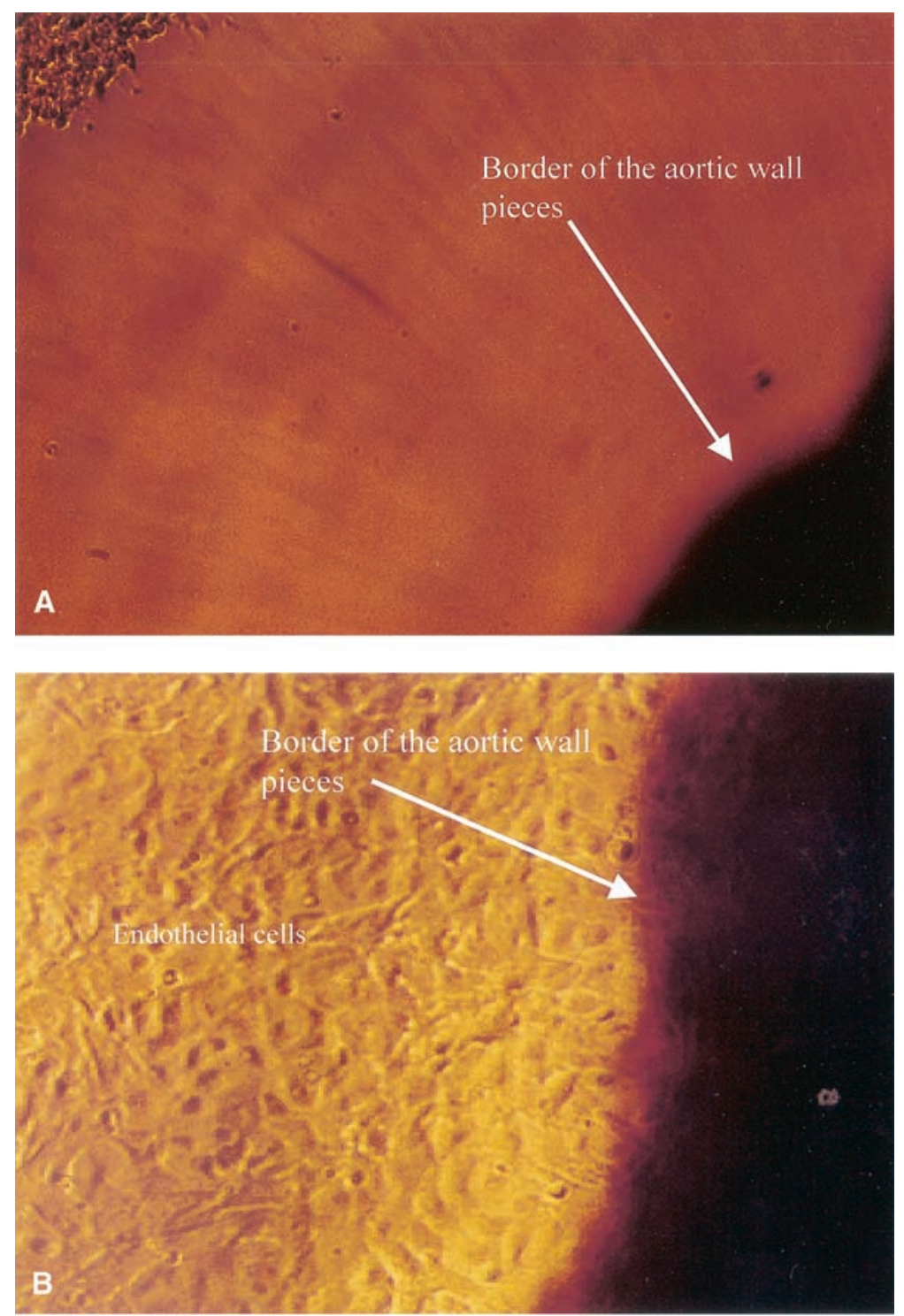

Figure 4. Toxicity testing. A, Arrows indicate margin of glutaraldehyde-fixed aortic wall piece that was only washed in physiologic sodium chloride solution for $\mathbf{1 0}$ minutes. Surrounding wall piece, no viable ECs are seen. B, Aortic wall piece pretreated with $\mathbf{1 0} \%$ citric acid. Wall piece lies directly on still confluent cell layer. There is no evidence of cell death.

hours (group B1) were still surrounded by a confluent EC layer after 72 hours of incubation with no evidence of further cell death. Aortic wall pieces pretreated with M-199 incubation and $5 \%$ or $10 \%$ citric acid also did not induce any cell death after 72 hours (Figure 4, B). Aortic wall pieces of group E1 (15\% citric acid) did not clearly induce cell death; the confluent cell layer, however, was somewhat dispersed.

\section{Seeding on Valve-containing Aortic Roots}

EC seeding. Isolated EC seeding on group 1 valves resulted in isolated, rounded up ECs on the luminal surface directly after the seeding procedure. After 7 days no ECs were seen on the leaflets or on the free wall.

Group 2 valves seeded with ECs had small islands of ECs scattered within the luminal surface of the valves. Initial cell attachment was $24 \%$. At 7, 14, and 21 days after the seeding procedure, although ECs were seen under scanning electron microscopy they did not reach confluence. The mean covered areas of the luminal surface were $30 \%$, $35 \%$, and $35 \%$ after 7,14 , and 21 days, respectively. Immunohistochemical staining for factor VIII proved some of these cells to be viable. On the bottoms of the culture dishes, ECs had grown to confluence after 7 days. 


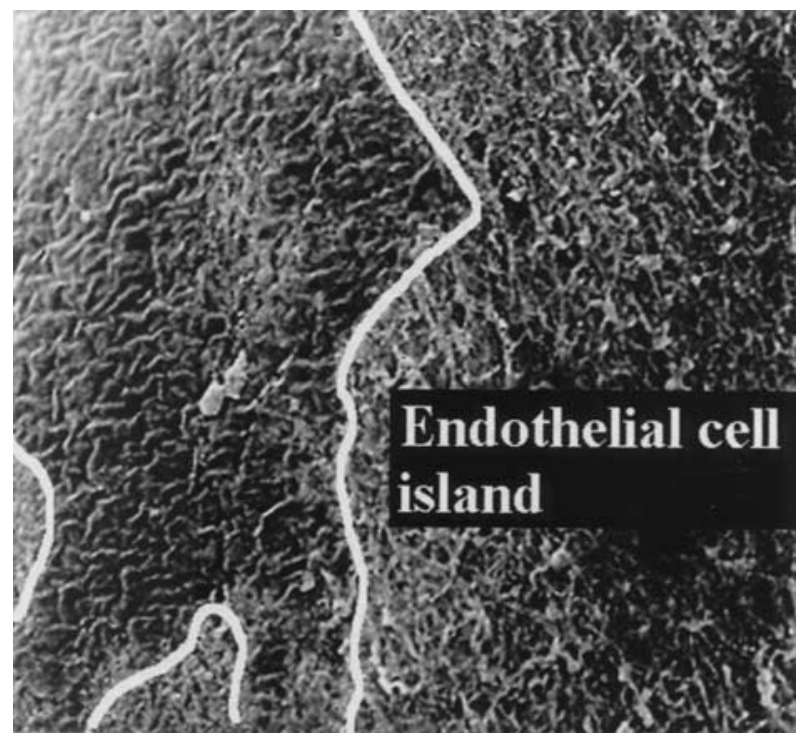

Figure 5. Islands of viable ECs on group C valves 7 days after seeding procedure. Arrows indicate margin of EC layer (scanning electron microscopy, original magnification $100 \times$ ).

On group 3 valves ECs also formed only islands upon the surface (Figure 5). Initial attachment was $45 \%$, but the mean covered areas were only $35 \%, 45 \%$, and $65 \%$ after 7,14 , and 21 days, respectively. Immunohistochemical staining for factor VIII and CD31 also proved the cells to be viable. A confluent or nearly confluent cell layer, however, was not seen on any of the valves.

EC seeding after preseeding with autologous fibroblasts. On group 1 valves fibroblast attachment was $45 \%$. EC seeding resulted in an attachment of $26 \%$. Under scanning electron microscopy islands of cell layers were seen, scattered over the whole luminal surface. Mean confluence was $25 \%$ after 7,14 , and 21 days. Immunohistochemical staining for factor VIII and CD31 proved the cells to be viable.

Fibroblast seeding on group 2 valves resulted in an attachment of $70 \%$. EC seeding on these valves had an initial attachment of $72 \%$. The mean surface areas covered by ECs were $80 \%, 90 \%$, and $95 \%$ after 7, 14, and 21 days, respectively. In one of these valves a confluent EC layer was seen 14 days after EC seeding; this layer remained stable after 21 days. The cells were proved viable by immunohistochemical stainings and grew to confluence on the bottom of the culture dish.

On group 3 valves fibroblast attachment was $78 \%$ (Figure 6). EC seeding resulted in an initial attachment of $76 \%$. Directly after seeding, the ECs formed a nearly confluent cell layer. Both valves had confluent EC layers at 7, 14, and 21 days after the seeding procedure (Figure 7). Immunohistochemical staining for factor VIII and CD31 proved the cells to be viable. The cell layer also stained positively for collagen IV (Figure 8, $A$ and $B$ ).

Seeding on commercially available porcine aortic valves. Corresponding to the previous results, these valves had pretreatment including 24 hours of incubation in M-199 followed by incubation with $10 \%$ citric acid and washing with distilled water until a neutral $\mathrm{pH}$ was reached. The valves were also preseeded with autologous fibroblasts, with an initial attachment of $76 \%$. EC seeding resulted in a nearly confluent cell layer directly after the seeding procedure with an initial attachment of $78 \%$. The cells grew to confluence after 7 days under culture conditions. The confluent cell layers remained stable for 21 days, and the cells stained positively for factor VIII and CD31 on the free wall as well as on the leaflets (Figure 9, $A$ and $B$ ). The luminal surface also stained positively for collagen IV. The confluent cell layer reached nearly to the polyester cloth covering. On this artificial surface itself, however, no ECs were found.

\section{Discussion}

Seeding of human ECs on biologic valve prostheses has been reported by several authors. ${ }^{5-12,16-20}$ The results, however, remained poor when glutaraldehyde-fixed valves were subjected to cell seeding. Continuing releases of toxic glutaraldehyde and remaining free aldehyde groups were assumed to be the major reasons. ${ }^{8-12}$ Although initial seeding on extensively rinsed valves was successful, ${ }^{8}$ the cells did not survive 7 days but rounded up and lost adhesion within 2 days. This agrees with the results of toxicity testing in our studies. Glutaraldehyde-fixed aortic wall pieces caused cell death of surrounding cultured human ECs. After incubation with buffered cell medium for 24 hours, however, the aortic wall pieces no longer showed cytotoxicity. Although fixation with complete cross-linking takes 7 days, unbound glutaraldehyde can be washed out more quickly, because when glutaraldehyde cross-links with collagen fibers it is bound covalently. In this state it is no longer reactive and cannot simply be washed out. Therefore only the unbound glutaraldehyde overhang, much less than the initial amount, must be washed out. However, the obvious loss of cytotoxicity indicated that no glutaraldehyde was released by the tissue.

Although the valvular leaflets are of greater clinical interest, we used aortic wall pieces for the toxicity testing. We did so because the free aortic wall has a smooth surface. This facilitated the comparison of the results of EC seeding, whereas the wavy surface of isolated leaflets proved to be more problematic in this setting. The conclusion from these experiments was that the release of unbound glutaraldehyde from the valves could be avoided by extensive rinsing with buffered solutions.

Although initial adhesion of human ECs on rinsed valves was successful, the cells did not survive longer than 3 days. ${ }^{8}$ After implantation in a primate model, the complete cell 


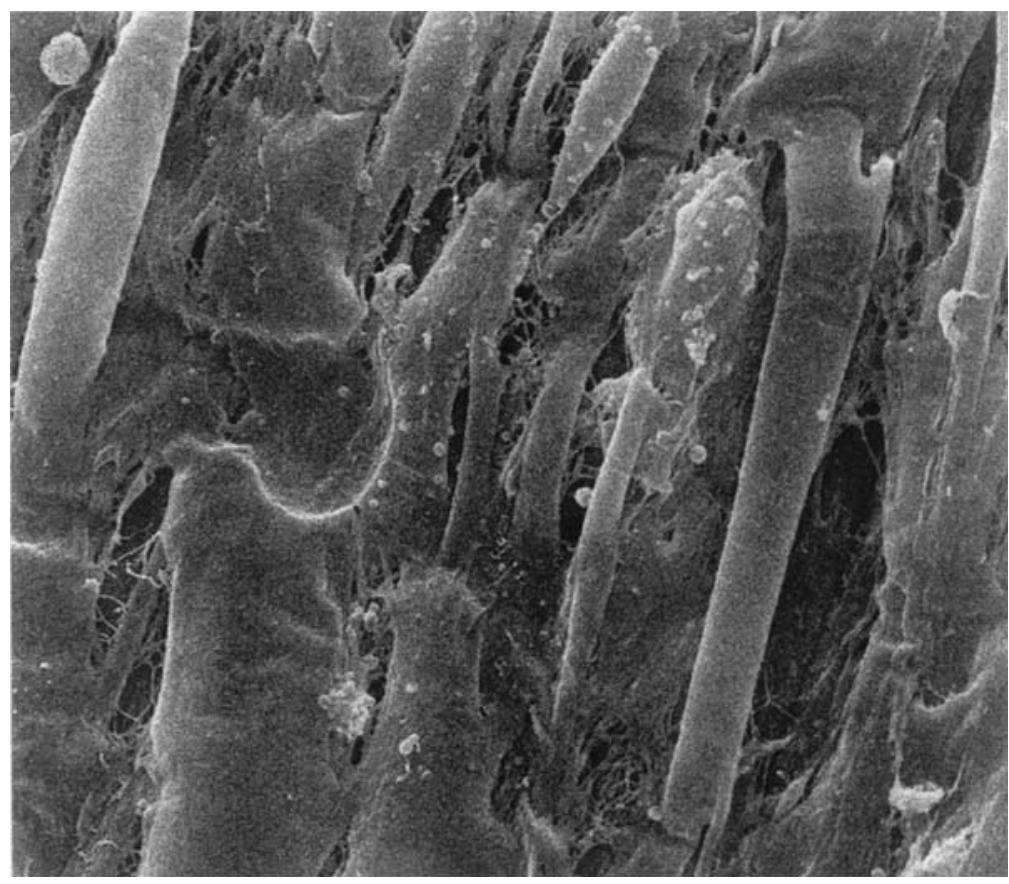

Figure 6. Fibroblasts seeded on group C valves (scanning electron microscopy, original magnification $1000 \times$ ).

layer was lost under in vivo conditions. ${ }^{10}$ Glutaraldehyde fixing cross-links collagen fibers by way of the amino groups of the proteins. These cross-links are initiated with the formation of a Schiff base between an amino group and glutaraldehyde (Figure 10). This product then reacts with another amino group to form a Schiff base. Alternatively, it reacts $^{21}$ according to aldol condensation (Figure 10). These reactions are responsible for the reduced immunogenicity of the prostheses and for their increased stiffness relative to fresh porcine aortic valves. The cross-links also, however, result in a hydrophobic surface, thus reducing the capability of cells to adhere. The pretreatment with $\alpha$-amino oleic acid used for the Medtronic Freestyle prosthesis decreases tissue calcification $^{22}$ and improves durability of the valves. The $\alpha$-amino oleic acid reacts with free aldehyde groups, thus reducing the cytotoxicity of the tissue. However, it further reduces hydrophilia of the surface by inducing large hydrophobic aliphatic chains into the collagen fibers. Cells attach to a surface by pseudopodia that use noncovalent bonds. This primary attachment therefore requires a hydrophilic surface. Cross-linking of the collagen fibers resulting from glutaraldehyde-fixation reduces hydrophilia of the surface (Figure 10). Our hypothesis was that the poor cell attachment to glutaraldehyde-fixed surfaces was caused by these cross-links rather than by the toxicity of the aldehyde. This hypothesis was supported by the results of the toxicity testing in this study. The adhesion of fibroblasts on these valves was better than that of ECs, although fibroblasts did not reach confluence either. Fibroblasts obviously had a

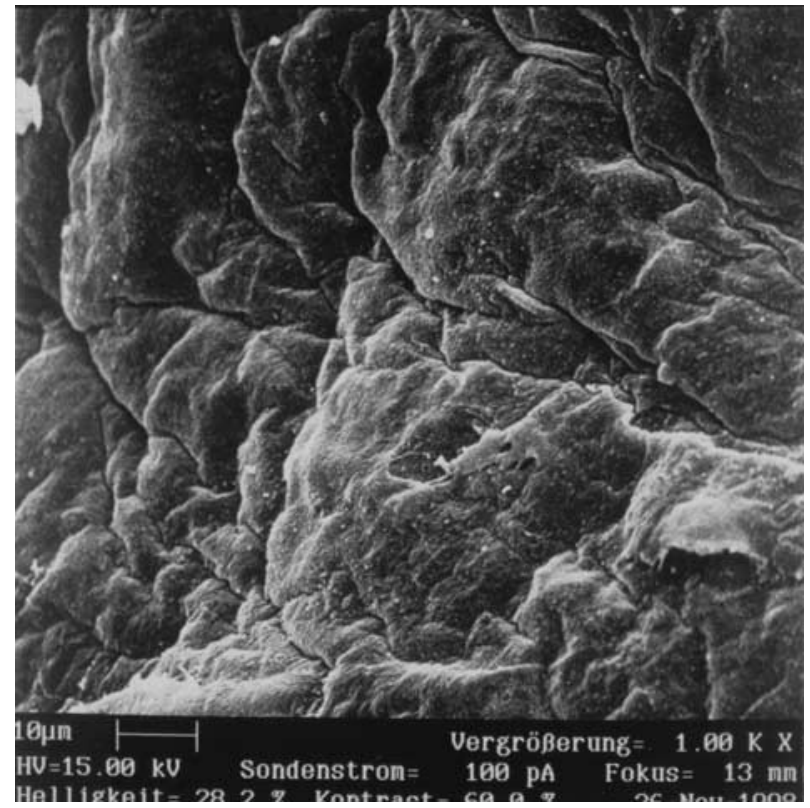

Figure 7. Confluent EC layer on group C valves preseeded with autologous fibroblasts (scanning electron microscopy, original magnification $1000 \times$ ).

better ability to adhere on different surfaces than did ECs. Additionally, their potential to build intercellular matrix proteins further improved their adhesion.

Because fibroblasts did not form confluent cell layers, we decided that an additional treatment of the valves was 

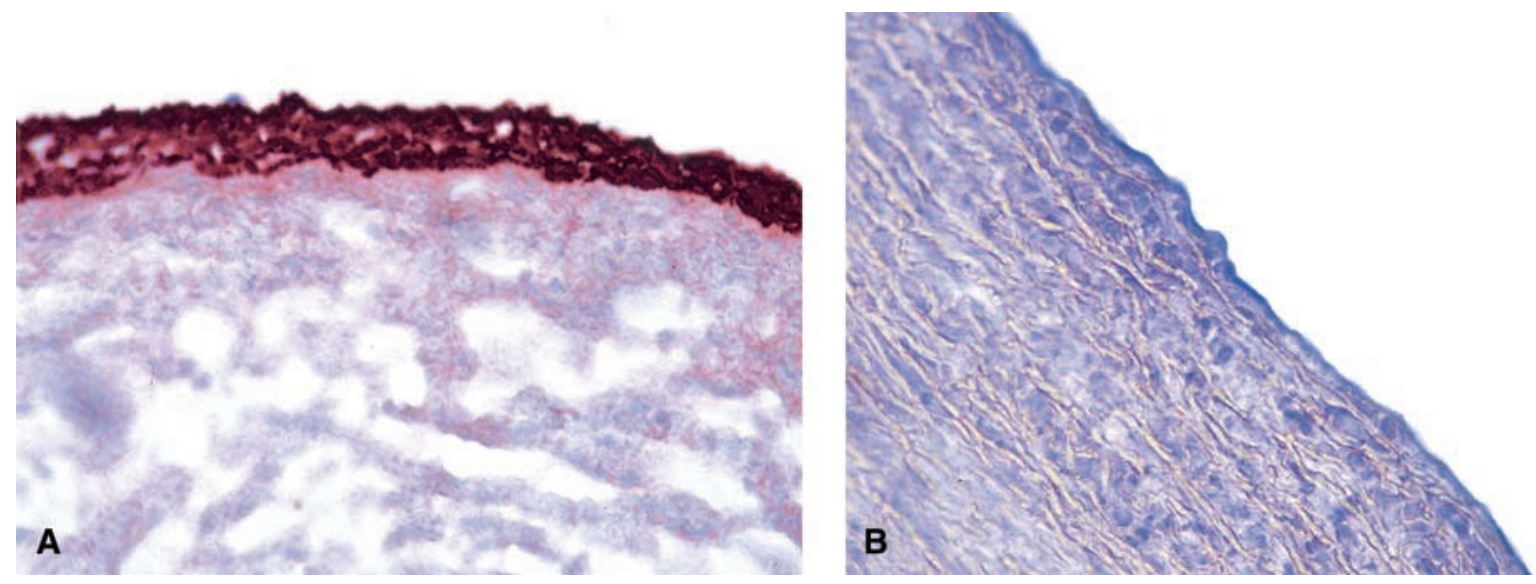

Figure 8. Immunohistochemical staining for collagen IV of group C valves after seeding with fibroblasts and ECs. A, Specimen clearly are stained positively for collagen IV at luminal surface, as indicated by red peroxidase reaction. B, Negative control. Small cell layer (stained blue) is seen, but no positive peroxidase reaction is found.
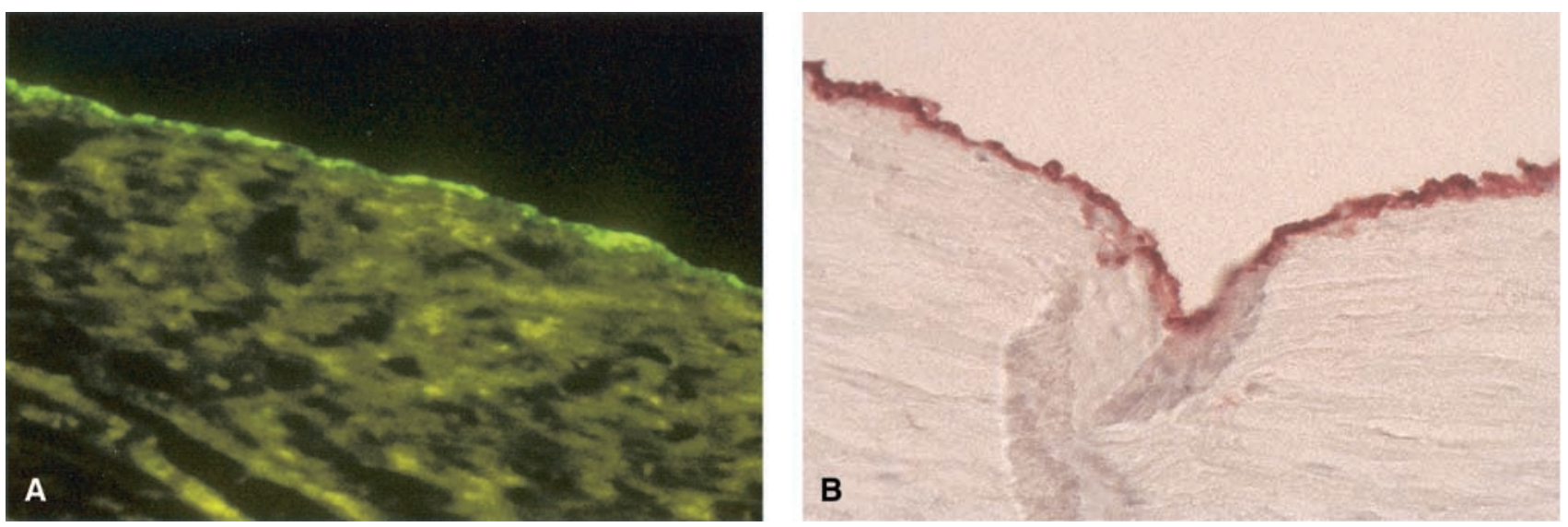

Figure 9. A, Immunohistochemical staining with factor VIII antibody and counterstaining with fluorescein of leaflets of commercially available porcine heart valve 21 days after cell seeding. Cells on surface are stained positively for factor VIII (fluorescing green), proving them to be viable ECs. B, Immunohistochemical staining with CD31antibody and peroxidase reaction of leaflets of commercially available porcine heart valve 21 days after cell seeding. Cells on surface are stained positively for CD31 (stained red).

necessary. Pretreatment with different amino acids was reported to improve cellular adhesion on glutaraldehyde-fixed valves. ${ }^{3-11}$ Incubation with an acidic $\mathrm{pH}$ particularly improved the success of cell seeding. ${ }^{9}$ We chose citric acid, a strong organic acid with four carboxyl-groups that binds to amino groups of the collagen fibers under salification (Figure 10). This strongly increases hydrophilia of the surface, thus enhancing cellular attachment. The pretreatment markedly improved cellular adhesion after cell seeding. EC seeding on such pretreated valves resulted in much higher attachment than seen on untreated valves. Therefore the pretreatment clearly had changed the surface properties to improve cellular adhesion. Seeding of ECs alone, however, did not result in a complete confluent cell layer. Successful
EC seeding on photo-oxidized bioprostheses, acellular matrix, and decellularized valves has been reported by several groups. ${ }^{16-20}$ Photo-oxidation avoids extensive cross-linking of collagen fibers but enhances hydrophilic properties through the oxidation process. Explanted valves showed tissue degeneration but no inflammatory response. ${ }^{21}$ The success of cell seeding on these xenogenic tissue valves supports our hypothesis that hydrophilia is an important factor for cell seeding. Seeding on unfixed but decellularized valves ${ }^{19}$ was successful, indicating the ability of ECs to attach to cell-free surfaces.

Despite the disadvantages of the fixation procedure, we chose glutaraldehyde-fixed valves as scaffolds for tissue engineering. The manufacturing of these prostheses is al- 

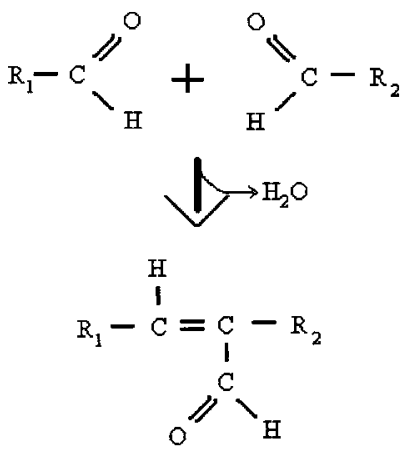

Aldol-Condensation

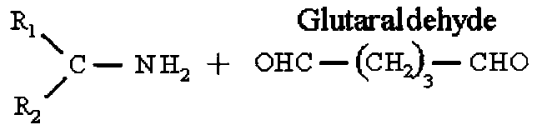<smiles>CC1CC1[15CH2]O</smiles><smiles>[R]C([R])N=CCCCC=O</smiles>

Schiff base

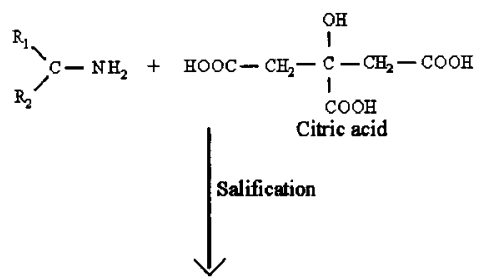

${ }_{\mathrm{R}_{2}}^{\mathrm{R}_{1}} \mathrm{C}^{\prime}-\mathrm{NH}_{3}^{+}+. \mathrm{OOC}-\mathrm{CH}_{2}-\stackrel{\mathrm{I}}{\mathrm{C}} \underset{\mathrm{COOH}}{\mathrm{I}}-\mathrm{CH}_{2}-\mathrm{COOH}$

Figure 10. Aldol condensation is physiologic process in the cross-linking of collagen fibers. ${ }^{21}$ Glutaraldehyde forms Schiff base with free amino groups of collagen fibers. Cross-linking can take place either as formation of another Schiff base with amino group of second fiber or by aldol condensation. Result, however, is rather hydrophobic. Citric acid (strong organic acid with four hydroxy groups) salifies amino groups, thereby inducing hydrophilia on surface of collagen fiber.

ready standardized, the glutaraldehyde-induced cross-linking reduces immunogenicity, and the fixation process facilitates surgical handling. If surface covering of these valves by ECs could delay tissue degeneration, these advantages would be of great importance for clinical use. Although ECs attach to many surfaces, the intensity of adhesion and subsequent stability of the cell layer depend mainly on the synthesis of extracellular matrix proteins. ECs synthesize collagen IV, an important part of the basal membranes ${ }^{21}$ of blood vessels. However, not all matrix proteins are produced by ECs themselves. Vascular fibroblasts also participate in the production of extracellular matrix. Fibroblast seeding on citric acid-pretreated valves showed high attachment rates. ECs seeded on these preseeded valves attached in large numbers and formed a confluent cell layer. Because fibroblasts obtained from different tissues differ in the types of collagen that they build, we used vascular fibroblasts for preseeding. This also may have contributed to the improved endothelial adhesion of these cells. Additionally, they maintained their physiologic synthesis of extracellular matrix, as was proven by the positive results of staining for collagen IV. This collagen type is synthesized physiologically only by $\mathrm{ECs}^{21}$ and is of great importance for the building of basal membranes. The synthesis of extracellular matrix proteins and components of the basal membrane is important in cellular adhesion and potential shear stress resistance. On the basis of the positive results of staining for these proteins, we hypothesize that the cell layer will be resistant to physiologic shear stress. However, further experimental studies are necessary, including in vitro shear stress testing.

Before such endothelialized valves can be used in clinical routine, in vivo experiments are necessary to prove whether the EC layer improves valve durability. To examine any impact of complete endothelialization, animal experiments are necessary. A potential influence on tissue calcification could be tested by implanting such aortic wall pieces subcutaneously in rats. Because the physical stress on the valvular structures also plays an important role in bioprosthetic degeneration, a large animal in vivo model must be used to test the complete valves under physiologic conditions. An additional benefit of a complete endothelialization might be a further reduction of thromboembolic events. However, the cell seeding procedure is costly. Therefore only an increase in durability would justify its use in the clinical routine. Graft selection could be done by the combination of echocardiography and electron beam tomography to chose the correct size of valve for endothelialization.

\section{Conclusions}

Pretreatment of porcine glutaraldehyde fixed aortic valves with an organic acid improved the results of cell seeding as a result of changes in the physicochemical properties of the valvular tissue. Preseeding with autologous fibroblasts resulted in a confluent and viable EC layer. 
We thank Dr. rer. nat. E. Gulbins for his support and advice regarding the chemical aspects of this paper.

\section{References}

1. Trantina-Yates AE, Human P, Bracher M, Zilla P. Mitigation of bioprosthetic heart valve degeneration through improved biocompatibility: in vitro versus spontaneous endothelialization. Biomaterials. 2001;22:1837-46.

2. Weissenstein C, Human P, Bezuidenhout D, Zilla P. Glutaraldehyde detoxification in addition to enhanced amine cross-linking dramatically reduces bioprosthesis calcification in the rat model. $J$ Heart Valve Dis. 2000;9:230-40.

3. Zilla P, Fullard L, Trescony P, Meinhart J, Bezuidenhout D, Gorlitze $\mathrm{D}$, et al. Glutaraldehyde detoxification of aortic wall tissue: a promising perspective for emerging bioprosthetic valve concepts. $J$ Heart Valve Dis. 1997;6:510-20.

4. Moritz A, Grimm M, Eybl E, Grabenwöger M, Windberger U, Dock $\mathrm{W}$, et al. Improved biocompatibility by postfixation treatment of aldehyde fixed bovine pericardium. ASAIO Trans. 1990;36:M300-3.

5. Bengtsson L, Radegran K, Haegerstrand A. In vitro endothelialization of commercially available heart valve bioprostheses with cultured adult human cells. Eur J Cardiothorac Surg. 1993;7:393-8.

6. Eberl T, Siedler S, Schumacher B, Zilla P, Schlaudraff K, Fasol R. Experimental in vitro endothelialization of cardiac valve leaflets. Ann Thorac Surg. 1992;53:487-92.

7. Fischlein T, Fasol R. In vitro endothelialization of bioprosthetic heart valves. J Heart Valve Dis. 1996;5:58-65.

8. Fischlein T, Lehner G, Lante W, Reichart B. Endothelialization of aldehyde-fixed cardiac valve bioprostheses. Transplant Proc. 1992; 24:2988.

9. Fischlein T, Lehner G, Lante W, Fittkau M, Murphy JG, Weinhold C, et al. Endothelialization of cardiac valve bioprostheses. Int J Artif Organs. 1994;17:345-52.

10. Lehner G, Fischlein T, Baretton G, Murphy JG, Reichart B. Endothelialized biological heart valves prostheses in the non-human primate model. Eur J Cardiothorac Surg. 1997;11:498-504.

11. Fu P, Lan H, Wang D, Guan H. Experimental study on modified treatment and endothelialization of bovine pericardial valves. $J$ Tongji Med Univ. 1997;17:136-9.
12. Hoffmann D, Gong G, Liao K, Macaluso F, Nikolic SD, Frater RW. Spontaneous host endothelial growth on bioprostheses. Circulation. 1992;89 Suppl 2:II75-9.

13. Haegerstrand A, Gillis C, Bengtsson L. Serial cultivation of adult human endothelium from the great saphenous vein. J Vasc Surg. 1992;16:280-5.

14. Sharefkin JB, Van Wart HE, Cruess DF, Albus RA, Levine EM. Adult human endothelial cell enzymatic harvesting: estimates of efficiency and comparison of crude and partially purified bacterial collagenase preparations by replicate microwell culture and fibronectin degradation measure by enzyme-linked immunosorbent assay. J Vasc Surg. 1986;4:567-77.

15. Terramani TT, Eton D, Bui PA, Wang Y, Weaver FA, Yu H. Human macrovascular endothelial cells: optimization of culture conditions. In Vitro Cell Dev Biol Anim. 2000;36:125-32.

16. Bengtsson LA, Philips R, Haegerstrand AN. In vitro endothelialization of photooxidatively stabilized xenogenic pericardium. Ann Thorac Surg. 1995;60(2 Suppl):S365-8.

17. Bengtsson L, Ragnarson B, Haegerstrand A. Lining of viable and nonviable allogenic and xenogenic cardiovascular tissue with cultured adult human venous endothelium. J Thorac Cardiovasc Surg. 1993; 106:434-43.

18. Jansson K, Bengtsson L, Swedenborg J, Hagerstrand A. In vitro endothelialization of bioprosthetic heart valves provides a cell monolayer with proliferative capacities and resistance to pulsatile flow. J Thorac Cardiovasc Surg. 2001;121:108-15.

19. Wilson GJ, Courtman DW, Klement P, Lee JM, Yeger H. Acellular matrix: a biomaterials approach for coronary artery bypass and heart valve replacement. Ann Thorac Surg. 1995;60(2 Suppl):S353-8.

20. Bader A, Schilling T, Teebken OE, Brandes G, Herden T, Steinhoff G, et al. Tissue engineering of heart valves - human endothelial cell seeding of detergent acellularized porcine valves. Eur J Cardiothorac Surg. 1998; 14:279-84.

21. Darnell J, Lodish H, Baltimore D. Molecular cell biology. 2nd ed. Scientific American Books. p. 906-15.

22. Girardot MN, Torrianni M, Girardot JM. Effect of AOA on glutaraldehyde-fixed bioprosthetic heart valve cusps and walls: binding and calcification studies. Int J Artif Organs. 1994;17:76-82.

23. Schoen FJ. Pathologic findings in explanted clinical bioprosthetic valves fabricated from photooxidized bovine pericardium. $J$ Heart Valve Dis. 1998;7:174-9. 